\title{
Temperature Variations on the Surface of the Si-star CU Virginis
}

\author{
T.A. Ryabchikova \\ Astronomical Council of the USSR Academy of Sciences, Moscow, USSR
}

\begin{abstract}
A hot region with an increase of effective temperature of $700 \mathrm{~K}$ was found on the surface of $\mathrm{Si}$-star CU Vir, based on the analysis of the available photometric and spectroscopic observations. This hot region is located near the phase of negative magnetic extremum and can explain both hydrogen line and infrared light variations.
\end{abstract}

The surface abundance inhomogeneity of magnetic CP stars is a well-established phenomenon. But in all investigations of chemical abundance and/or spotty structure of magnetic CP (CP2) stars the effective temperature is considered to be constant over the surface although there are some indications of regions with different temperature on the surface of CP2 stars. To explain the observed amplitudes of photometric uvby variations and energy distribution at different phases in the $\mathrm{Si}$ star CU Vir, Weiss et al. (1976) suggested $\Delta T_{\text {eff }} \sim 600 \mathrm{~K}$, as well as $\Delta \log g \sim 0.15$, at phases of maximum and minimum light. In this paper we present arguments for temperature variations on the surface of CU Vir, based on the analysis of the available photometric and spectral data.

$\mathrm{CU}$ Vir $=\mathrm{HD} 124224$ is the Si-star with the shortest known rotational period among CP stars (0d52). This star has been studied photometrically by many authors who give practically the same period of light variations. In this paper we used the ephemeris from Blanco and Catalano (1971): $J D$ (lightmin.) = $2439995.4413+0.52067688 E$.

The existence of a phase shift $\Delta \phi \sim 0.15$ between the visible light curves and the $\mathrm{H} \gamma$ intensity variations (Ryabchikova, 1972) did not permit us to explain the latter by-temperature variations, although $\mathrm{H} \gamma$ and $\mathrm{H} \beta$ line profile variations in the case of CU Vir are indicative of a temperature rather than gravity effect (Musielok et al., 1990). In order to reconcile photometric and spectroscopic observations of CU Vir we analysed the variations of different parameters available from the literature. We used photometry of CU Vir taken from White et al. (1980) and Pyper and Adelman (1985). Intensity variations of He I $\lambda 4026$ are taken from electrophotometric measurements by Pedersen (1978) and are converted into equivalent widths using a relation proposed in his paper. Equivalent width variations of Si II $\lambda 3862$ are taken from Krivosheina et al. (1980), those of $\mathrm{H} \beta$ from Musielok et al. (1990). $\beta$-index measurements by White et al. (1980) and Pyper 
and Adelman (1985) are converted into equivalent widths through the relation: $W(\mathrm{H} \beta)=-19.58+37.857(\beta-2.00)($ Osawa, 1965$) . \mathrm{H} \gamma$ radial velocity measurements are taken from Abt and Snowdon (1973) and magnetic field measurements are taken from Landstreet and Borra (1977).

First of all we consider the phase correspondence between the hydrogen line variations and the colour index $[u-b]$ calculated from White et al. (1980) and Pyper and Adelman (1985). This index has been shown to be the most reliable temperature indicator for Si-stars (Mégessier, 1988). From Fig. 1 one can see a good phase agreement between $W(\mathrm{H} \beta)$ and $[u-b]$. Using the Mégessier (1988) calibration we estimate two values of effective temperature: $T_{\text {eff }}=12000 \mathrm{~K}$ (phases $0.05-0.15$ ) and $T_{\text {eff }}=12700 \mathrm{~K}$ (phases $0.6-0.7$ ). This temperature difference agrees well with that obtained from IR observations (Catalano and Kroll, private communication). A model with a hot region near the phase 0.6 explains both the equivalent width and the radial velocity variations of hydrogen lines. To get a better reproduction of $\mathrm{H} \beta$ and $\mathrm{H} \gamma$ amplitudes we must accept a small difference in gravity, $\Delta \log g \sim 0.2$, at phases 0.1 and 0.6 . The resulting temperature and gravity differences are in good agreement with those of Weiss et al. (1976), but the position of the hot region is shifted in phase compared with theirs.

In Fig. 2 we present the visual light variations, Si II $\lambda 3862$ and He I $\lambda 4026$ equivalent width variations and variations of effective magnetic field. One can see that regions of different effective temperature roughly coincide with the magnetic field extrema. We performed calculations of line intensities at phases 0.1 and 0.6 using the "ABSTAR" program (Ryabchikova and Piskunov, 1988) with the corresponding Kurucz (1979) models.

We obtained the following results: 1 . Near phase $0.1 \mathrm{CU}$ Vir looks like a star with nearly normal helium and silicon abundance. 2. Temperature rise near phase 0.6 can fully explain the secondary maximum of $\mathrm{He}$ I line intensity variation, as well as a small depletion in Si II line intensity variation.

The double wave structure of visual light variations can be easily explained by joint effect of enhanced silicon opacity near phase 0.35 and of the temperature increase near phases $0.6-0.7$. If the silicon and helium anomalies are caused by a diffusion process then we must conclude that this process operates near the magnetic equator.

\section{References}

Abt, H.A., Snowdon, M.S.: 1973, Astrophys. J. Suppl. 25, 137

Blanco, C., Catalano, F.: 1971, Astron. J. 76, 630

Catalano, F., Kroll, R.: private communication

Krivosheina, A.A., Ryabchikova, T.A., Khokhlova, V.L.: 1980, Nautch. Inf. 47, 70

Kurucz, R.L.: 1979, Astrophys. J. Suppl. Ser. 40, 1

Landstreet, J.D., Borra, E.F.: 1977, Astrophys. J. (Letters) 212, L46

Mégessier, C.: 1988, Astron. Astrophys. Suppl. Ser. 72, 551

Musielok, B., Ryabchikova, T.A., Davydova, E.S., Madej, J.: 1990, Mitt. Karl-Schwarzschild Obs. Tautenburg, Nr. 125, 110 


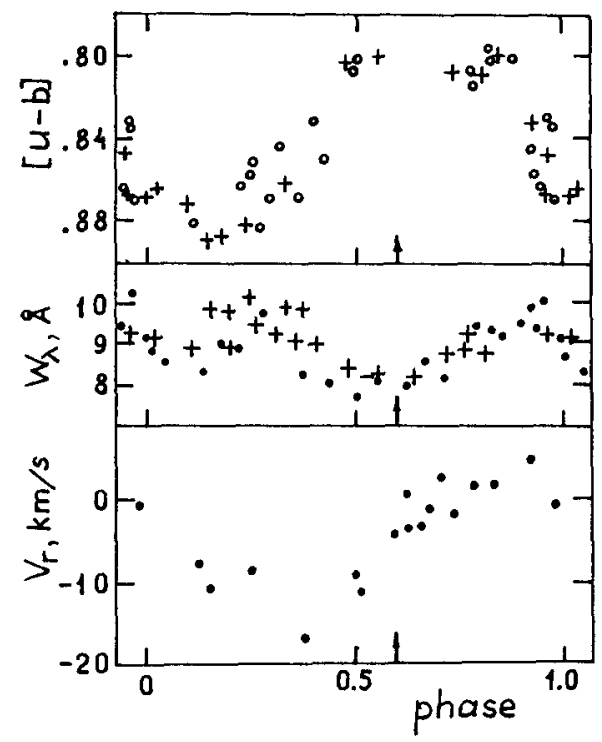

Fig. 1. Variations of index $[u-b](a)$, $\mathrm{H} \beta$ equivalent width (b), and $\mathrm{H} \gamma$ radial velocity (c). a. open circles - data from White et al. (1980), crosses - data from Pyper and Adelman (1985). b. dots data from Musielok et al. (1990). c. dots data from Abt and Snowdon (1973). Position of the hot region is indicated by arrows.

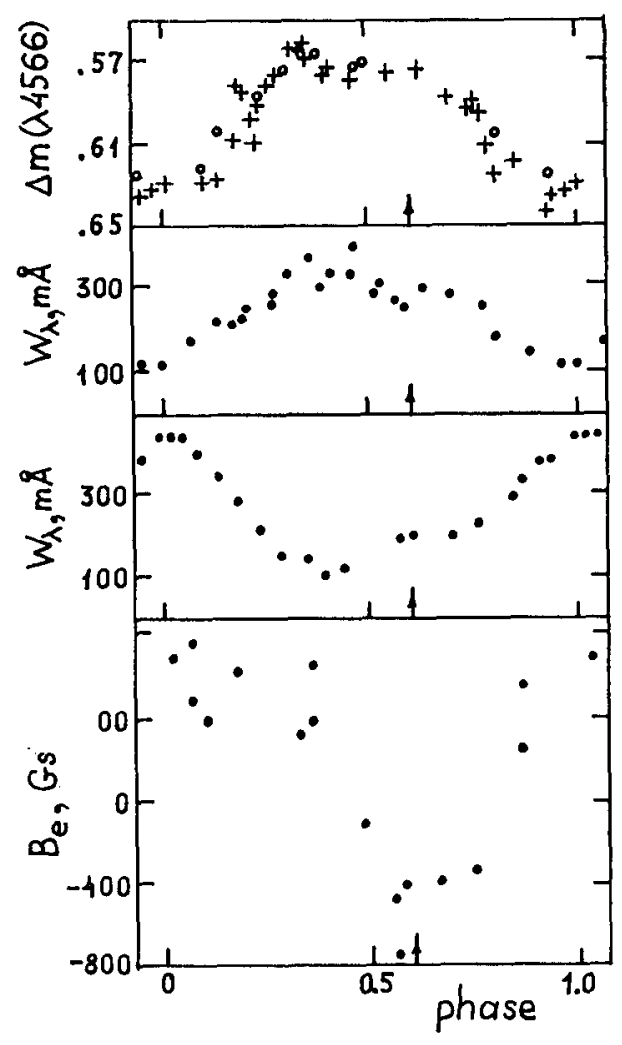

Fig. 2. Variations of visual light (a), equivalent widths of Si II $\lambda 3862$ (b) and He I $\lambda 4026$ (c) lines and effective magnetic field (d). For symbols on (a) see Fig. 1. The position of the hot region is indicated by the arrows.

Osawa, K.: 1965, Ann. Tokyo Astron. Obs. 9, 123

Pedersen, H.: 1978, Astron. Astrophys. Suppl. Ser. 33, 203

Pyper, D.M., Adelman, S.J.: 1985, Astron. Astrophys. Suppl. Ser. 59, 369

Ryabchikova, T.A.: 1972, Izv. Krymsk. Astrofiz. Obs. 45, 146

Ryabchikova, T.A., Piskunov, N.E.: 1988, in Elemental Abundance Analysis, eds. S.J. Adelman, T. Lanz, Univers. Lausanne, p. 93

White, R.E., Pyper, D.M., Adelman, S.J.: 1980, Astron. J. 85, 836

Weiss, W.W., Albrecht,R., Wieder, R.: 1976, Astron. Astrophys. 47, 423 